\title{
A tennis-based health program for middle-aged men who are at risk for heart disease
}

\author{
Flavio Rosa Jr ${ }^{1}$, Juan Pedro Fuentes ${ }^{2}$, Aline Pertile Remor ${ }^{1}$, Karina Ghisoni ${ }^{1}$, Paulo César Lock Silveira ${ }^{1}$, Vitor Costa ${ }^{3}$, Rafael Luiz Prim ${ }^{4}$, \\ Débora da Luz Scheffer ${ }^{1}$, Aderbal Silva Aguiar Jr ${ }^{1}$, Luiz Guilherme Antonacci Guglielmo ${ }^{3}$, and Alexandra Latini ${ }^{1 *}$ \\ ${ }^{1}$ Bioenergetics and Oxidative Stress Laboratory - LABOX, Federal University of Santa Catarina, Florianopolis, Brazil \\ ${ }^{2}$ Sport Sciences Faculty, University of Extremadura, Cáceres, Spain \\ ${ }^{3}$ Physical Effort Laboratory - LAEF, Federal University of Santa Catarina, Florianópolis, Brazil \\ ${ }^{4}$ Clinical Analysis Laboratory, Hospital Professor Polydoro Ernani de São Thiago, Florianópolis, Brazil
}

\begin{abstract}
The purpose of this study was to investigate the health effects of an adapted tennis-based exercise program, in middle-aged men with moderate risk of cardiovascular diseases (CVD). Fifteen men ( $44.9 \pm 4.9$ years) with two or more CVD risk factors, completed an eight-week health program that included a continuous tennisbased exercise protocol with constant and controlled intensity between 70\%-85\% of maximum heart rate and a standard nutritional orientation. Participants were assessed at the beginning and end of the exercise program for standard parameters related to CVD and the Framingham risk score, motor skills and cardiopulmonary fitness, peripheral inflammation (neopterin levels), oxidative stress (nitric oxide and carbonylated protein content), and mitochondrial function (lymphocyte complex $\mathrm{I}$ activity). Exercise improved muscle strength and power, speed, agility and flexibility, $\dot{\mathrm{V}}_{2}$ max and metabolic equivalents. The participants experienced decreases in body mass index, waist circumference, total and LDL-cholesterol, and reduction in the Framingham Risk Score. Exercise also decreased peripheral oxidative stress and production of nitric oxide. In addition, the increased $\mathrm{VO}_{2}$ at ventilatory threshold 1 correlated with enhanced mitochondrial activity in blood lymphocytes. The tennis-based exercise program adapted to sustaining moderate and constant intensity represents a valuable non-pharmacological intervention to prevent CVD.
\end{abstract}

\section{Introduction}

Cardiovascular diseases (CVD) are a major cause of mortality [1], while physical exercise reduces mortality from all causes [2]. A healthy lifestyle - including regular physical activity, a balanced diet, and weight management - is the best way to prevent and treat CVD [3]. The American College of Sports Medicine (ACSM) recommends that most adults engage in moderate-intensity cardiorespiratory exercise training for $\geq 30 \mathrm{~min} /$ day $^{-1}$ on $\geq 5$ days. week $^{-1}$, or a vigorousintensity cardiorespiratory exercise training for $\geq 20$ min.day $^{-1}$ on $\geq 3$ days.week ${ }^{-1}$, or a combination of both [4]. Adults should also perform resistance exercises for each of the major muscle groups, and neuromuscular exercises involving balance, agility, and coordination on a 2-3 days.week ${ }^{-1}$ basis, according to ACSM [4].

According to the ACSM's recommendations [4], a large number of studies demonstrate significant improvements in CVD risk factors in overweight and/or sedentary individuals [5]. Keep yourself physically active has been shown to be a successful strategy in preventing CVD. Many studies have focused on traditional exercise approaches, i.e., running, walking, resistance training or a combination of those. The effectiveness of more complex models of exercise, such as sports, still lacks evidence. Moreover, many people do not enjoy walking or running, which results in low adherence to physical activity [6]. In addition, it might be necessary to practice more than one of these exercises to improve physical and mental health, and specifically to reduce CVD risk factors including enhanced exercise capacity, muscular strength, and reduced body fat mass [7]. Sports might be a good option to promote good health, since most of them require complex physical functions, such as motor coordination, agility and speed, muscle power and endurance, among others. Outdoor sports are motivating and enjoyable with high adherence levels [6].

Tennis is a sport that improves motivation, strength and muscular endurance, recovering capacity and motor coordination [8]. A tennis match satisfies the ACSM's recommendations [4] for quantity and quality of exercise involved for the development and maintenance of cardiovascular fitness in healthy adults, independent of the individual's tennis proficiency [9]. Evidence indicates that regular tennis training decreases cardiovascular risk factors in healthy subjects $[9,10]$. Middleaged tennis players displayed an improved $\mathrm{VO}_{2}$ max, decreased body fat mass, and greater grip strength [11]. Thus, tennis sport modality can be suggested as an attractive alternative to health orientated sports programs $[9,10,12]$. In agreement, a recent published cohort performed in a Scottish and English population showed a robust inverse association between participation in racquet sports and and mortality, with a substantial reductions in all-cause and CVD mortality [13]. Although, there is little evidence of the molecular benefits of these programs for subjects at risk for heart diseases. The purpose of this study was to investigate the effects of an adapted tennis-based exercise

Correspondence to: Alexandra Latini, Laboratório de Bioenergética e Estresse Oxidativo - LABOX, Departamento de Bioquímica, Centro de Ciências Biológicas, Universidade Federal de Santa Catarina, Campus Universitário Córrego Grande, Bloco C-201/214, Florianópolis - SC, 88040-900, Brazil, Tel: +55 48 37214743; Fax: +55 483721 9672; E-mail: alatini@ccb.ufsc.br

Key words: Cardiovascular diseases, tennis, Framingham risk score, mitochondria Received: February 28, 2017; Accepted: March 27, 2017; Published: March 30, 2017 
program for health promotion in middle-aged sedentary men with moderate risk of CVD.

\section{Methods}

Fifteen sedentary middle-aged men (44.9 \pm 4.9 years), from Florianopolis, Santa Catarina, Brazil participated in the exercise program. The subjects did not meet the minimum needs of physical activity recommended by ACSM [4] in the previous 6 months to the program; thus, they were considered sedentary individuals. The participants presented one to four of the following CVD risk factors: family history of acute myocardial infarction, dyslipidemia, overweight/ obesity or hypertension. The baseline anthropometric, motor skills, body composition and anthropometric plasma lipid profile are described in Table 1. Clinical follow-up was performed on a weekly basis by a cardiologist, a psychologist and a nutritionist for the duration of the program. One subject received a beta blocker (propranolol), one subject received an angiotensin II receptor antagonist (losartan), two of them received antidepressants of the serotonin/norepinephrine reuptake inhibitor class (venlafaxine and paroxetine) and one subject received an alpha-adrenergic blocker for urinary retention associated with benign prostatic hyperplasia (doxazosin mesylate). All subjects were informed of the purpose and risks of the study, and provided informed written consent. The study protocol was approved by the local ethics committee (Comitê de Ética em Pesquisa com Seres Humanos - 846/11).

1. The tennis-based physical training protocol employed the following activities at the physiology exercise lab and the tennis court:

The incremental maximal cardiopulmonary test was performed on a horizontal treadmill starting at a speed of $4 \mathrm{~km} \cdot \mathrm{h}^{-1}$, increasing at $1 \mathrm{~km} \cdot \mathrm{h}^{-1} \cdot \mathrm{min}^{-1}$. Gas exchanges were measured using breath-bybreath equipment (Cosmed, Quark CPET, Rome, Italy), and the data was smoothed and averaged at 15-second intervals to measure heart rate (HR), peak oxygen consumption ( $\dot{\mathrm{V}}_{2}$ peak), minute ventilation

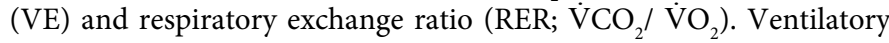
threshold (VT) 1 and 2 were identified according to Davis et al. [14].

2. The subjects performed motor skills tests and were also evaluated for anthropometric, physiological and cardiovascular risk measures as described below:
The physical training lasted 2 months, and it was performed 3 times.week ${ }^{-1}$ on alternate days (Mondays, Tuesdays, and Fridays) in an outdoor tennis court (Centro de Ciências do Desporto, Universidade Federal de Santa Catarina, Florianópolis, Brazil). Each $1 \mathrm{~h}$ exercise session was divided into $10 \mathrm{~min}$ of warm up, $40 \mathrm{~min}$ of moderateintensity training, and $10 \mathrm{~min}$ of cool down. Tennis exercise sessions were restlessly continuous in the individual $70-85 \%$ maximal heart rate $\left(\mathrm{HR}_{\max }\right)$. The $\mathrm{HR}$ was monitored and recorded at 5-second intervals during the exercise (Polar FT1, Kempele, Finland). Some modifications were included to control the exercise intensity $\left(70-85 \% \mathrm{HR}_{\max }\right)$ during the tennis sessions. In a traditional tennis lesson, the coach feeds a set of balls to one student at a time (Figure 1A, player 1), and the others passively recover at the corner of the court (Figure 1A, player 2-4). In the tennis-based physical training proposed by Garcia et al. [12], subjects are allowed to let the ball bounce twice at the beginning, playing mainly doubles rather than singles games (Figure 1B, players 1-2). In addition, four different on-field movements are performed simultaneously for players 3-6 while they are not involved in the tennis specific activities, in order to maintain the effort within the HR (Figure 1B, players 3-6). We adapted Garcias's program [12], as shown in Figure 1C, by adding extra motor skill activities which are performed around the court and allow the players to maintain exercise intensity. The activities consisted of running on an agility ladder, skipping drills, varied displacements with cones, and tennis strokes simulation with a medicine ball.

3. An educational nutrition program was conducted to change the subjects' lifestyle and to control coronary risk factors.

The physiological and cardiovascular risk measures involved rest blood pressure (BP) and HR and they were measured in the morning (8:00 AM) using the automatic Oregon, Scientific BPW120. The anthropometry measures body mass $(\mathrm{kg})$, height $(\mathrm{m})$, waist-tohip ratio (WHR, $\mathrm{cm}$ ), and skinfold thickness $(\mathrm{mm})$ were evaluated also for calculation of body fat percentage [15]. The cardiovascular risk measures included body mass index $\left(\mathrm{BMI}, \mathrm{kg} / \mathrm{m}^{2}\right)$, WHR, and Framingham risk score. Framingham risk score for men considers age, blood total- and HDL-cholesterol levels, smoking status and rest systolic BP [16].

Motor evaluation was performed in the physiology exercise lab. Lower limb flexibility was assessed with the traditional sit-and-reach

Table 1. Fitness measures, body composition and lipid profile at baseline (PRE) and after (POST) the tennis-based program.

\begin{tabular}{|c|c|c|c|c|c|c|c|}
\hline Fitness measures & PRE & POST & ES & Body and lipid profiles & PRE & POST & ES \\
\hline $\mathrm{VO}_{2 \text { peak }}\left(\mathrm{mL} \cdot \mathrm{kg}^{-1} \cdot \mathrm{min}^{-1}\right)$ & $36.8 \pm 5.8$ & $38.9 \pm 6.4^{*}$ & 0.34 & Body mass $(\mathrm{kg})$ & $95.1 \pm 12.0$ & $92.8 \pm 11.1^{*}$ & 0.20 \\
\hline MET & $10.50 \pm 1.65$ & $11.11 \pm 1.82^{*}$ & 0.35 & Lean body mass $(\mathrm{kg})$ & $63.6 \pm 6.8$ & $64.8 \pm 6.6$ & 0.18 \\
\hline Rest HR (bpm) & $70.7 \pm 10.8$ & $68.5 \pm 9.3$ & 0.22 & Fat body mass (kg) & $31.4 \pm 7.0$ & $27.9 \pm 6.0^{* * *}$ & 0.54 \\
\hline $\mathrm{HR}_{\max }(\mathrm{bpm})$ & $173.8 \pm 10.3$ & $173.1 \pm 10.7$ & 0.07 & Body mass index $\left(\mathrm{kg} \cdot \mathrm{m}^{-2}\right)$ & $31.3 \pm 5.2$ & $30.5 \pm 4.7^{*}$ & 0.16 \\
\hline $\mathrm{VE}_{\max }\left(\mathrm{L} \cdot \mathrm{min}^{-1}\right)$ & $110.3 \pm 18.7$ & $115.8 \pm 19.0^{\dagger}$ & 0.29 & Percentage of body fat (\%) & $32.8 \pm 4.2$ & $29.9 \pm 3.7^{* * *}$ & 0.73 \\
\hline HR at VT1 (bpm) & $114.0 \pm 13.6$ & $118.7 \pm 14.9$ & 0.33 & Waist circumference $(\mathrm{cm})$ & $102.5 \pm 11.0$ & $99.2 \pm 9.5^{* * *}$ & 0.32 \\
\hline HR at VT2 (bpm) & $151.9 \pm 12.1$ & $155.6 \pm 12.2^{\dagger}$ & 0.30 & Hip circumference $(\mathrm{cm})$ & $110.4 \pm 7.8$ & $108.7 \pm 6.8$ & 0.23 \\
\hline $\mathrm{VO}_{2}$ at VT1 $\left(\mathrm{mL} \cdot \mathrm{kg}^{-1} \cdot \mathrm{min}^{-1}\right)$ & $17.9 \pm 4.5$ & $20.4 \pm 6.9$ & 0.44 & Waist/hip ratio & $0.93 \pm 0.05$ & $0.91 \pm 0.05$ & 0.40 \\
\hline $\mathrm{VO}_{2}$ at VT2 $\left(\mathrm{mL} \cdot \mathrm{kg}^{-1} \cdot \mathrm{min}^{-1}\right)$ & $31.7 \pm 5.9$ & $34.6 \pm 6.5^{*}$ & 0.47 & Fast glucose & $87.3 \pm 13.3$ & $88.4 \pm 12.2$ & 0.09 \\
\hline Rest systolic BP (mmHg) & $132.07 \pm 15.8$ & $131.4 \pm 14.9$ & 0.04 & Triglycerides & $118.53 \pm 56.3$ & $124.47 \pm 62.1$ & 0.10 \\
\hline Rest diastolic BP (mmHg) & $90.2 \pm 14.0$ & $87.8 \pm 14.7$ & 0.17 & Total cholesterol & $219.8 \pm 65.4$ & $185.4 \pm 53.8^{*}$ & 0.58 \\
\hline $\operatorname{RER}\left(\mathrm{VCO}_{2} \cdot \mathrm{VO}_{2}^{-1}\right)$ & $1.10 \pm 0.09$ & $1.10 \pm 0.07$ & 0.00 & HDL-cholesterol & $48.9 \pm 7.5$ & $46.7 \pm 8.9$ & 0.27 \\
\hline Sit and reach $(\mathrm{cm})$ & $18.0 \pm 8.5$ & $22.2 \pm 7.4^{* * * *}$ & 0.53 & LDL-cholesterol & $152.2 \pm 64.7$ & $116.9 \pm 50.4^{*}$ & 0.61 \\
\hline Abdominal strength (rep. $\min ^{-1}$ ) & $24.3 \pm 10.6$ & $29.4 \pm 8.2^{* * * *}$ & 0.54 & \multirow{5}{*}{\multicolumn{4}{|c|}{$\begin{array}{l}\text { MET: metabolic equivalent. HF: heart frequency. VE: minute ventilation. } \mathrm{VO}_{2} \text { : oxygen } \\
\text { consumption. BP: blood pressure. VT1/2: ventilatory threshold } 1 / 2 \text {. RER: resting metabolic } \\
\text { rate. ES: effect size. ES: effect size. }{ }^{\dagger} P<0.10,{ }^{*} P<0.05 \text {, }{ }^{* * *} P<0.001 \text {. }\end{array}$}} \\
\hline Horizontal jump (m) & $1.68 \pm 0.22$ & $1.79 \pm 0.22^{* *}$ & 0.50 & & & & \\
\hline Medicine ball throw (m) & $6.95 \pm 0.96$ & $7.32 \pm 0.94^{*}$ & 0.39 & & & & \\
\hline 20-m sprint (s) & $3.85 \pm 0.26$ & $3.76 \pm 0.24^{*}$ & 0.36 & & & & \\
\hline Shuttle run test (s) & $8.21 \pm 0.46$ & $7.94 \pm 0.53^{* * * *}$ & 0.55 & & & & \\
\hline
\end{tabular}



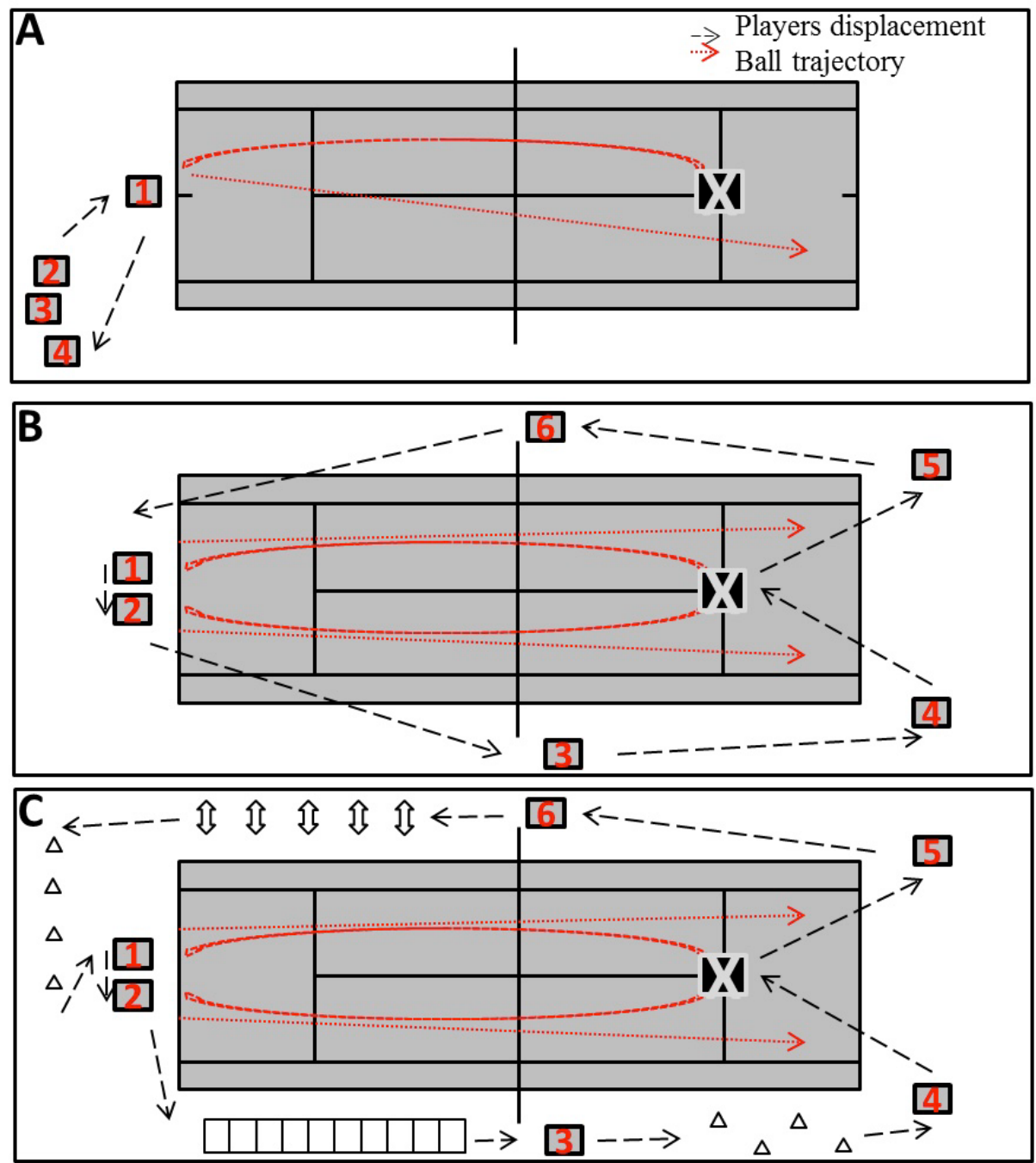

Figure 1. Court layout of a traditional tennis lesson, where the coach (indicated as X) feeds a set of balls to each student at the time (students are identified as numbers 1 to 4), whereas the others passively recover at the corner of the court (A); Disposal of an adapted tennis lesson proposed by Garcia et al.[12], in which the coach feeds two students at the same time while the others move around the court (running, walking; active interval) to pick up some balls and put back in the coach's basket, so that they keep exercise intensity between desired hear rate zone (B); Adapted tennis training with motor skills activities around the court during a coach centred activity (C).

test $(\mathrm{cm})$. Lower body muscle power was assessed with the horizontal jump test $(\mathrm{m})$ and upper body muscle power with the medicine ball $(2$ $\mathrm{kg}$ ) throw test $(\mathrm{m})$. Abdominal strength was assessed as a maximum number of repetitions during one min. The shuttle run test evaluated agility $(5 \times 5 \mathrm{~m})$. The sprint fitness test over 20 meters measured running speed and acceleration. Participants performed each test twice and the highest scores were considered.

A food frequency questionnaire was used to evaluate dietary habits before the health promotion program. Participants received nutritional orientation, according to the food pyramid of Brazilian Society of Functional Nutrition.

For the biochemical measurements $20 \mathrm{~mL}$ of blood were collected by venous puncture in $3.2 \%$ citrate-conditioned tubes. Ten $\mathrm{mL}$ were used for lymphocytes isolation according to Golzari et al. [17] and the final pellet was suspended in $4.4 \mathrm{mM}$ potassium phosphate buffer $\mathrm{pH}$ 7.4, containing $0.3 \mathrm{M}$ sucrose, $5 \mathrm{mM}$ MOPS, $1 \mathrm{mM}$ EGTA and $0.1 \%$ bovine serum albumin. This preparation was used to measure mitochondrial complex I activity. The other $10 \mathrm{~mL}$ of blood were centrifuged at $900 \times$ $\mathrm{g}$ for $20 \mathrm{~min}$ and the plasma was used for measuring the lipid profile, glucose levels, and the content of neopterin, nitrites and carbonylated proteins.

The lipid profile, total cholesterol, HDL-cholesterol, LDLcholesterol, triglycerides, and glucose were determined in the biochemical autoanalyser Dimension RxL (Siemens Healthcare Diagnostics Inc., EUA).

Neopterin concentrations were determined by high-performance liquid chromatography (HPLC) coupled to fluorescence detection as previously reported by our group [18-20], and values were expressed as $\mu$ mol. $\mathrm{L}^{-1}$. The oxidant status was assessed by measuring the content of carbonylated proteins based on the reaction with 2,4-dinitrophenylhydrazone, results were expressed as nmol of carbonyl groups per mg protein and calculated by using the extinction coefficient of $22,000 \times 10^{6} \mathrm{nmol} / \mathrm{mL}$ for aliphatic hydrazones. Nitric oxide formation was estimated spectrophotometrically as the content of nitrites and values were expressed and nmol.mL ${ }^{-1} \cdot \mathrm{mg}$ protein ${ }^{-1}$. Mitochondrial activity was assessed by measuring the activity of complex I based on the rate of $\mathrm{NADH}$-dependent ferricyanide reduction at $420 \mathrm{~nm}$ and results were expressed as nmol. $\mathrm{min}^{-1} \cdot \mathrm{mg}$ 
protein $^{-1}$. The enzyme activity was measured using a Varian Cary 50 spectrophotometer with temperature control (Varian Inc., Palo Alto, CA, USA). These methodologies were performed as previously reported by our group $[18,21]$.

Data is described as mean \pm standard error of the mean (SEM). The normally distributed variables were analysed using the paired Student $\mathrm{t}$ test and non-normally distributed variables using the Wilcoxon rank test. Effect sizes (ES) were calculated. ES values $>0.2$ indicate a small change; $>0.5$ a moderate change; and $>0.8$ a large A P value $<0.05$ was considered to be statistically significant.

\section{Results}

The 8-week tennis program improved various fitness measures in the subjects shown in Table 1 . The effect size was small $(E S>0.2)$ for $\dot{\mathrm{V}} \mathrm{O}_{2}$ peak $\left[\mathrm{t}_{(13)}=2.62 ; \mathrm{P}<0.05\right]$, MET $\left[\mathrm{t}_{(13)}=2.78 ; \mathrm{P}<0.05\right], \dot{\mathrm{VO}}_{2}$ at $\mathrm{VT} 2$ $\left[\mathrm{t}_{(13)}=2.19 ; \mathrm{P}<0.05\right]$, medicine ball throw $\left(\mathrm{t}_{(14)}=2.33 ; \mathrm{P}<0.05\right)$ and the $20 \mathrm{~m}$ sprint $\left(\mathrm{t}_{(14)}=2.38 ; \mathrm{P}<0.05\right)$. However, the effect size was moderate $(\mathrm{ES}>0.5)$ for lower limb flexibility $\left[\mathrm{t}_{(13)}=6.25 ; \mathrm{P}<0.001\right]$, abdominal strength $\left[\mathrm{t}_{(13)}=4.46 ; \mathrm{P}<0.001\right]$, horizontal jump [ $\left.\mathrm{t}_{(14)}=3.54 ; \mathrm{P}<0.01\right]$ and the shuttle run test $\left[\mathrm{z}_{(13)}=3.30 ; \mathrm{P}<0.001\right]$. No significant changes were observed in the rest $\mathrm{HR}$ and $\mathrm{HR}_{\max }, \mathrm{BP}$, maximum VE, HR, VT1 and $\mathrm{VT} 2, \dot{\mathrm{VO}}$, at VT1, and RER.

Exercise also improved the body composition and the lipid profile of the participants. As shown in Table 1, the effect size of exercise was small (ES $>0.2)$ for body mass $\left[\mathrm{t}_{(14)}=2.81 ; \mathrm{P}<0.05\right]$, WHR $\left[\mathrm{t}_{(14)}=3.45\right.$; $\mathrm{P}<0.01]$ and HDL-cholesterol levels $\left[\mathrm{t}_{(14)}=2.21 ; \mathrm{P}<0.05\right.$ ]; but, it was moderate $(E S>0.5)$ for fat mass $\left[\mathrm{t}_{(14)}=6.40 ; \mathrm{P}<0.001\right]$, percentage of body fat $\left[\mathrm{t}_{(14)}=3.28 ; \mathrm{P}<0.01\right]$, and the total cholesterol levels $\left[\mathrm{t}_{(14)}=2.18\right.$; $\mathrm{P}<0.05]$

Exercise reduced plasma carbonylated protein content $\left[\mathrm{t}_{(9)}=7.23\right.$; $\mathrm{P}<0.001 ; \mathrm{ES}=3.8]$, improved plasma NO levels $\left[\mathrm{z}_{(9)}=1.98 ; \mathrm{P}<0.05\right.$; ES $>0.8]$, and increased mitochondrial activity of complex I in blood lymphocytes $\left[\mathrm{t}_{(2)}=9.19 ; \mathrm{P}=0.01 ; \mathrm{ES}=7.54\right]$ (Figure 2A, B and D; Panel I). The mitochondrial activity of complex I in lymphocytes positively correlated with $\dot{\mathrm{V}} \mathrm{O}_{2}(\mathrm{~mL} / \mathrm{kg} / \mathrm{min})$ at $\mathrm{VT} 1\left[\mathrm{r}^{2}=0.66, \mathrm{P}<0.05\right]$. No changes were observed in plasma neopterin levels. Finally, all these changes decreased Framingham risk score $\left[\mathrm{z}_{(14)}=2.25 ; \mathrm{P}<0.05\right.$; Figure 2E; Panel II] and the percentage risk of developing coronary heart disease $\left[\mathrm{z}_{(14)}=2.06 ; \mathrm{P}<0.05\right.$; Figure 2F; Panel II].

\section{Discussion}

The present study proposes a new approach for physical activities linked to health programs that aim to prevent primary CVDs. The exercise protocol applied was based on the modified tennis training method described initially by Garcia et al. [12]. In our adapted protocol, tennis training was performed at a constant intensity between $70 \%$ and $85 \%$ of $\mathrm{HR}_{\max }$, and various exercises were included around the tennis court in order to maintain the $\mathrm{HR}_{\max }$, and to improve the motor skill performance. As a positive outcome of this novel protocol, participants enhanced agility, speed, flexibility, muscle power and abdominal strength that resulted in improved fitness, body composition and lipid profile and decreased plasmatic oxidative stress. Altogether, the

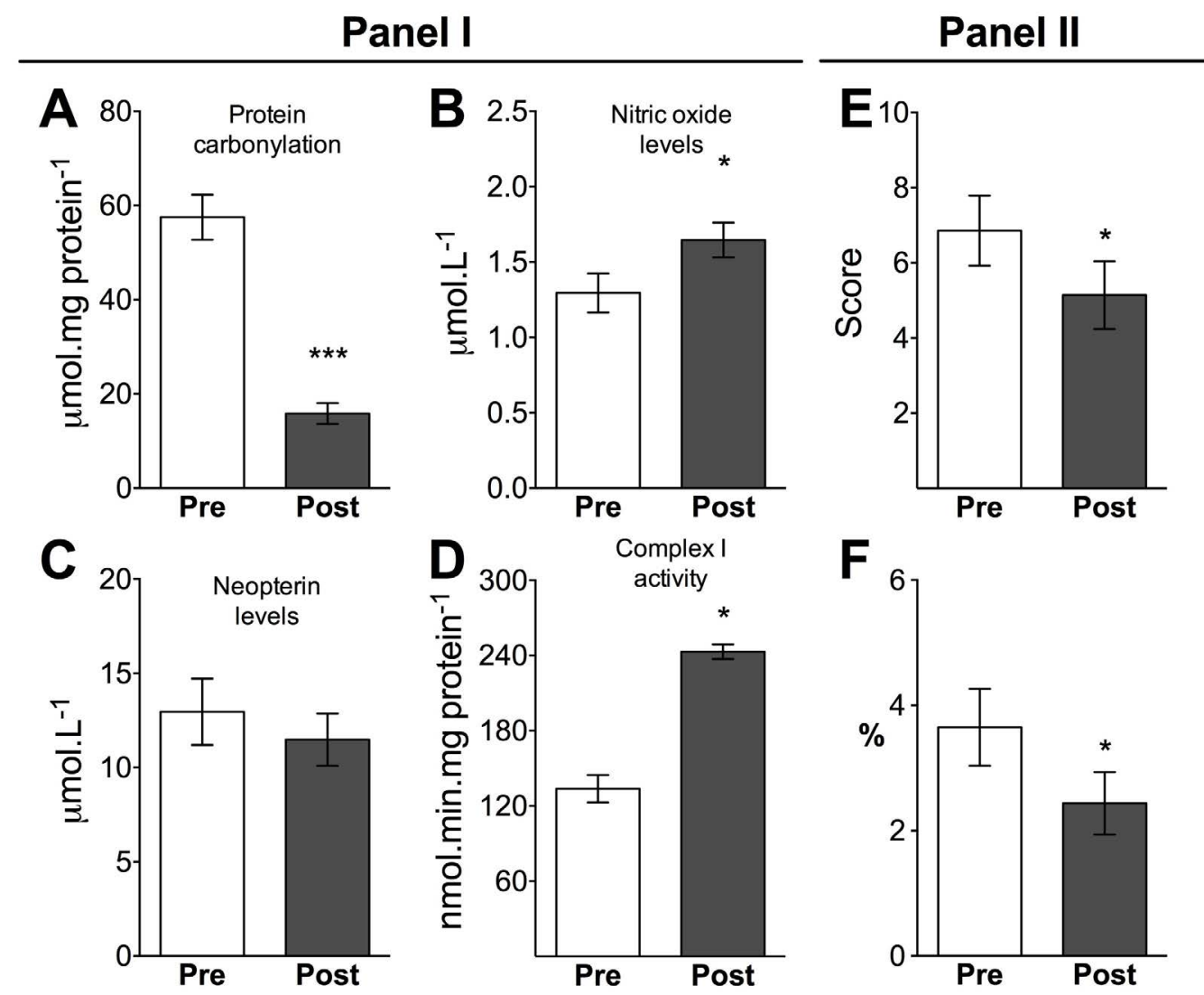

Figure 2. Panel I. Plasma biomarkers of protein oxidative damage (A), nitric oxide (NO, B), inflammation (neopterin levels; C) and lymphocyte mitochondrial activity (D) at baseline (PRE) and after (POST) the tennis-based program. Panel II. Framingham risk score (E) and risk of heart disease (F) at baseline (PRE) and after (POST) the tennis-based program. Results are expressed in mean \pm SEM. $* P<0.05 ; * * * P<0.001$ (paired Student $t$ test). 
exercise protocol decreased the Framingham risk score (the index that estimates the risk of heart attack in the next 10 years).

Improved fitness is strongly associated with a reduction in the risk of developing coronary heart disease, and a reduction in mortality risk $[2,3]$. Moreover, the improvements in strength, agility and flexibility promote better performance in activities of daily living [4], positively impacting the participant's quality of life.

Exercise programs generally have a duration of 12 weeks $[4,22]$ and include walking programs, walking/running exercise on treadmills [23] or aerobic training and/or resistance trainings [22,23]. Interestingly, those studies with groups of aerobic training, resistance training or the combination of both, only found significant results similar to our study for combined aerobic and resistance training exercise groups, such as reduction of body weight, body fat, $\mathrm{BMI}$ and $\mathrm{V}_{2}$ peak $[7,24]$. This means that our exercise protocol with a sport activity induced the same benefits as the practice of more than one exercise type combined.

A similar study to the one presented here was conducted by Ferrauti et al. [10] in 16 middle-aged healthy recreational tennis players and the authors also found positive outcomes for aerobic power and body composition; however, the tennis protocol applied was not strictly monitored and mainly consisted of hitting a tennis ball and running afterwards. Therefore, it could not be concluded at the end of the program, whether the risk for CVDs was reduced.

Exercise capacity is an important prognostic factor in subjects with CVD risk, with both reduced $\dot{\mathrm{VO}}_{2}$ max and reduced exercise time during the stress test being powerful predictors of mortality [12]. The capacity of enhancing 1 MET increases survival rates by $12 \%$ [25]. That is, a single environmental change such as beginning the practice of playing tennis will have a great impact on general health, including the cardiovascular system.

We also demonstrated here that the 8-week tennis training improved mitochondrial activity in peripheral lymphocytes, which correlated significantly with $\dot{\mathrm{VO}}_{2}$ at VT1 (the onset of plasma lactate accumulation) [26]. Mitochondria are essential organelles engaged mainly in the production of ATP through oxidative metabolism. Measuring mitochondrial activity in blood samples could be used as a marker of metabolic capacity or mitochondrial oxidative power, as previously demonstrated after endurance training in skeletal muscle biopsies in healthy subjects and athletes $[18,27]$. Since lymphocyte mitochondria complex I activity correlated positively to the percentage of $\dot{\mathrm{VO}}{ }_{2}$ peak at VT1 (and this parameter is similar to the onset of plasma lactate accumulation [26]) it may be assumed that these mitochondrial adaptations occurred also in muscle fibers. Thus, when the activity of complex I increase, there is also an increase in treadmill velocity and percentage of $\dot{\mathrm{V}}_{2}$ peak that lactate accumulates due to heavy exercise.

The exercise program also induced positive effects in the blood lipid profiles of sedentary middle-aged men, particularly a decrease in total cholesterol and LDL-cholesterol levels (Table 1). The study from Saremi et al. [28], which applied a walking/running program for 12 weeks, found similar results for total cholesterol and LDL-cholesterol. Garcia and coworkers [12] also found improvements in lipid profile after an adapted tennis program for secondary prevention of acute myocardium infarction, such as a reduction in total cholesterol, LDLcholesterol and triglycerides, and also an increase in HDL-cholesterol. On the other hand, the study by Ferrauti and colleagues [10] in which tennis exercise intensity was not individually controlled, did not achieve the benefits in reducing total or LDL-cholesterol concentrations, but did improve HDL-cholesterol levels. Evidence shows that continuous aerobic exercise is efficient in improving HDL-cholesterol levels [4].

These enhancements may also have been related to changes in diet participants reduced the consumption of fat, including fried food, fast food and milk, along with a significant increase in the consumption of high-fiber foods (data not shown), which could have contributed to the reduction of total cholesterol as previously demonstrated [4], since it is known that high intake of trans fatty acids has a direct proinflammatory effect that is associated with a higher risk of CVD.

We measured neopterin as an inflammation parameter, since this metabolite reflects the activation of the immune system and the establishment of an inflammation state. Although, the reduction of neopterin levels have been associated with reduction in CVD risk [29], we did not observed changes in this measurement, but we did observe a significant decrease in plasma carbonylated proteins after the tennis training. This reduction in peripheral oxidant stress may be associated with an exercise-induced up-regulation of the antioxidant system [30]. Increased plasma protein carbonyl content has been associated with higher CVD risk, myocardial infarction and mortality [31].

We also observed improved NO levels after the 8-week tennis program. NO failure, synthesis and signalling are related to atherogenesis and risk of death. NO plays a crucial vascular role in endothelial relaxation and vasodilatation, and with lower CVD risk, as previously reported [32]. There is a strong link between NO production and vascular/endothelial integrity and functionality. Therefore, the increased NO levels induced by the exercise protocol promoted vascular/endothelium health in the participants.

\section{Conclusions}

This sport-based program for good health promotion focused on primary prevention of CVDs, in which exercise intensity was controlled during all exercise sessions. Several CVD-related parameters were improved, including exercise capacity, BMI, body fat, WHR, muscle strength, lipid profile, oxidative stress, NO levels, mitochondrial activity, and the Framingham risk score. These results strongly corroborate the literature to recommend physical activity for heart disease prevention. Finally, the inclusion of a sport modality in health promotion programs might increase the adhesion of the participants and represent a valuable non-pharmacological intervention to prevent primary CVDs.

\section{Practical implications}

- A tennis-based exercise program adapted to sustaining moderate and constant intensity represents a valuable lifestyle modification to prevent primary CVDs in middle-aged subjects.

- The adapted tennis program improved fitness, body composition, plasma lipid profile, oxidative stress and vascular activity, and reduced the Framingham risk score in middleaged subjects at risk for heart diseases.

- The tennis-based exercise program improved aerobic (maximal oxygen consumption, $\dot{\mathrm{V}} \mathrm{O}_{2} \max$ ) and anaerobic (lactate threshold) fitness indexes in middle-aged subjects.

- The tennis training program increased mitochondrial activity in peripheral lymphocytes, which correlated significantly with $\dot{\mathrm{VO}}_{2}$ at VT1 (the onset of plasma lactate accumulation), suggesting this measure could be used as a marker of muscle metabolic capacity or mitochondrial oxidative power. 


\section{Acknowledgements}

The authors are grateful to Theodore Griswold for language editing. This work was supported by grants from $\mathrm{CNPq}$ (Conselho Nacional de Desenvolvimento Científico e Tecnológico; 479222/20134) and CAPES (Coordenação de Aperfeiçoamento de Pessoal de Nível Superior; 004_2013). Latini A is a CNPq fellow (311451/2013-6).

\section{Conflict of interest}

Rosa Jr F, Fuentes JP, Remor AP, Ghisoni K, Silveira P, Costa V, Prim RL, Guglielmo LGA, Aguiar Jr A, and Latini A declare that they have no conflict of interest.

\section{References}

1. World Health Organization (2010) Global status report on noncommunicable diseases. Geneva, Switzerland: World Health Organization, pp: 176

2. Autenrieth CS, Baumert J, Baumeister SE, Fischer B, Peters A, et al. (2011) Association between domains of physical activity and all-cause, cardiovascular and cancer mortality. Eur J Epidemiol 26:91-99.

3. Nandish S, Wyatt J, Bailon O, Smith M, Oliveros R, et al. (2011) Implementing cardiovascular risk reduction in patients with cardiovascular disease and diabetes mellitus. Am J Cardiol 108: 42B-51B. [Crossref]

4. Garber CE, Blissmer B, Deschenes MR, Franklin BA, Lamonte MJ, et al. (2011) American College of Sports Medicine position stand. Quantity and quality of exercise for developing and maintaining cardiorespiratory, musculoskeletal, and neuromotor fitness in apparently healthy adults: guidance for prescribing exercise. Med Sci Sports Exerc 43:1334-1359.

5. Yusuf S, Hawken S, Ounpuu S, Dans T, Avezum A, et al. (2004) Effect of potentially modifiable risk factors associated with myocardial infarction in 52 countries (the INTERHEART study): case-control study. Lancet 364:937-952.

6. Raedeke TD (2007) The relationship between enjoyment and affective responses to exercise. Journal of Applied Sport Psychology 19:105-115.

7. Willis LH, Slentz CA, Bateman LA, Shields AT, Piner LW, et al. (1985)Effects of aerobic and/or resistance training on body mass and fat mass in overweight or obese adults. J Appl Physiol 113:1831-1817.

8. Gucciardi DF, Jackson B, Hanton S, Reid M (2015) Motivational correlates of mentally tough behaviours in tennis. J Sci Med Sport 18: 67-71.[Crossref]

9. Fernandez-Fernandez J, Sanz-Rivas D, Sanchez-Munoz C, Pluim BM, Tiemessen I, et al. (2009) A comparison of the activity profile and physiological demands between advanced and recreational veteran tennis players. J Strength Cond Res 23:604-610.

10. Ferrauti A, Weber K, Strüder HK (1997) Effects of tennis training on lipid metabolism and lipoproteins in recreational players. Br J Sports Med 31: 322-327. [Crossref]

11. Vodak PA, Savin WM, Haskell WL, Wood PD (1980) Physiological profile of middleaged male and female tennis players. Med Sci Sports Exerc 12:159-163.

12. Garcia JP, Giraldo VM, Barrado JJ, Casasola CD (2013) Tennis training sessions as a rehabilitation instrument for patients after acute myocardial infarction. $J$ Sports Sci Med 12:316-322.

13. Oja P, Kelly P, Pedisic Z, Titze S, Bauman A, et al. (2016) Associations of specific types of sports and exercise with all-cause and cardiovascular-disease mortality: a cohort study of 80306 British adults. Br J Sports Med. [Crossref]

14. Davis JA, VodakP, Wilmore JH, Vodak J, Kurtz P (1976) Anaerobic threshold and maximal aerobic power for three modes of exercise. J Appl Physiol 41:544-550.
15. VasconcelosFde A, Cordeiro BA, Rech CR, Petroski EL (2010) Sensitivity and specificity of the body mass index for the diagnosis of overweight/obesity in elderly. Cad Saude Publica 26: 1519-1527.[Crossref]

16. D'Agostino RB Sr, Vasan RS, Pencina MJ, Wolf PA, Cobain M, et al. (2008) Genera cardiovascular risk profile for use in primary care: the Framingham Heart Study. Circulation 117: 743-753. [Crossref]

17. Golzari Z, Shabkhiz F, Soudi S, Kordi MR, Hashemi SM (2010) Combined exercise training reduces IFN-gamma and IL-17 levels in the plasma and the supernatant of peripheral blood mononuclear cells in women with multiple sclerosis. Int Immunopharmacol 10:1415-1419.

18. de Lucas RD, Caputo F, Mendes de Souza K, Sigwalt AR, Ghisoni K, et al. (2014) Increased platelet oxidative metabolism, blood oxidative stress and neopterin levels after ultra-endurance exercise. J Sports Sci 32:22-30.

19. Ghisoni K, Latini A, Kuehne LK, ReiberH, Bechter K, et al. (2015) Cerebrospinal fluid neopterin is brain-derived and not associated with blood-CSF barrier dysfunction in non-inflammatory affective and schizophrenic spectrum disorders. $J$ Psychiatr Res 63:141-142.

20. Ghisoni K, Martins Rde P, Barbeito L, Latini A (2015) Neopterin as a potential cytoprotective brain molecule. J Psychiatr Res 71: 134-139. [Crossref]

21. Silveira PC, SchefferDda L, Glaser V, Remor AP, Pinho RA, et al. (2016) Low-leve laser therapy attenuates the acute inflammatory response induced by muscle traumatic injury. Free Radic Res 50:503-513.

22. Ho SS, Dhaliwal SS, Hills AP, Pal S (2012) The effect of 12 weeks of aerobic, resistance or combination exercise training on cardiovascular risk factors in the overweight and obese in a randomized trial. BMC Public Health 12: 704. [Crossref]

23. Morris CK, Froelicher VF (1993) Cardiovascular benefits of improved exercise capacity. Sports Med 16: 225-236. [Crossref]

24. American College of Sports Medicine Position Stand (1998) The recommended quantity and quality of exercise for developing and maintaining cardiorespiratory and muscular fitness, and flexibility in healthy adults. Med Sci Sports Exerc 30:975-991.

25. Perez AB (2008) Exercise as the cornerstone of cardiovascular prevention. Revista Espanola De Cardiologia 61:514-528.

26. Farrell PA, Wilmore JH, Coyle EF, Billing JE, Costill DL (1993) Plasma lactate accumulation and distance running performance. 1979. Med Sci Sports Exerc 25:10911097.

27. Sparks LM, Johannsen NM, Church TS, Earnest CP, Moonen-Kornips E, et al. (2013) Nine months of combined training improves ex vivo skeletal muscle metabolism in individuals with type 2 diabetes. J Clin Endocrinol Metab 98:1694-1702.

28. Saremi A, Shavandi N, Parastesh M, Daneshmand H (2010) Twelve-week aerobic training decreases chemerin level and improves cardiometabolic risk factors in overweight and obese men. Asian J Sports Med 1:151-158.

29. Grammer TB, Fuchs D, Boehm BO, Winkelmann BR, Maerz W (2009) Neopterin as a predictor of total and cardiovascular mortality in individuals undergoing angiography in the Ludwigshafen Risk and Cardiovascular Health study. Clin Chem 55:1135-1146.

30. Aguiar AS Jr., Pinho RA (2010) The Brain Redox Paradox of Physical Exercise. In: Kozyrev D, Slutsky V, editors. Handbook of Free Radicals: Formation, Types and Effects. 1. 1 ed. New York: Nova Sicence, pp: 153-166.

31. Leopold JA, Loscalzo J (2009) Oxidative risk for atherothrombotic cardiovascular disease. Free RadicBiol Med 47: 1673-1706. [Crossref]

32. Napoli C, de Nigris F, Williams-Ignarro S, Pignalosa O, Sica V, et al. (2006) Nitric oxide and atherosclerosis: an update. Nitric Oxide 15: 265-279. [Crossref]

Copyright: (C2017 Rosa F. This is an open-access article distributed under the terms of the Creative Commons Attribution License, which permits unrestricted use, distribution, and reproduction in any medium, provided the original author and source are credited. 